\title{
Use of the Vestibular Disorders Activities of Daily Living Scale to describe functional limitations in patients with vestibular disorders ${ }^{1}$
}

\author{
Helen S. Cohen \\ Bobby R Alford Department of Otolaryngology - Head and Neck Surgery, Baylor College of Medicine, One Baylor \\ Plaza, Houston, TX 77030, USA \\ Tel.: +1 713798 6336; Fax: +1 713798 8658; E-mail: hcohen@bcm.edu
}

Received 27 August 2012

Accepted 18 December 2012

\begin{abstract}
The Vestibular Disorders Activities of Daily Living Scale (VADL) is one of several self-rated scales in the literature that may be useful for determining level of functional limitation or disability in people with vestibular disorders. The VADL was designed by an occupational therapist for use in treatment planning during vestibular rehabilitation. Unlike many other scales the VADL is specifically focused on essential functional skills and important mobility and instrumental skills. This paper reviews the findings about the VADL, including the original research about its development and more recent papers using it. The scale is most useful for assessing the patient's perception about independence in personal, self care and basic mobility skills. It provides an outline for discussing instrumental activities of daily living.
\end{abstract}

Keywords: Quality of life, self care, limitation of activity, vertigo, dizziness, occupational therapy, physical therapy

Patients with vestibular disorders have decreased independence in self-care and mobility skills [4]. Their functional limitations vary somewhat across disorders. For example many patients with Meniere's disease are independent during quiescent periods but have limited functional skills during periods of Meniere's attacks or more active disease [5]. Even patients with benign paroxysmal positional vertigo (BPPV), who some clinicians consider to be unimpaired, report specific deficits in activities and skills that involve pitch rotations of the head [6]. The standard scales of functional performance, such as the Klein-Bell [19] or the Barthel Index [21], are not sensitive to the subtle problems ex-

\footnotetext{
${ }^{1}$ This paper was presented at the Session: Sensory Transduction at the 8th Symposium on the Role of the Vestibular Organs in Space Exploration, April 8-10, 2011, Houston, TX, USA.
}

perienced by people with vestibular impairments. The Vestibular Disorders Activities of Daily Living scale (VADL) was developed by the author, an occupational therapist, for use in evaluation and treatment planning by therapists who provide vestibular rehabilitation. The scale was developed to fill the gap between the existing scales and the clinical need.

Before the VADL was published the literature included only two scales of quality of life that were specific to vestibular disorders, the UCLA Dizziness Questionnaire (UCLA-DQ) and the Dizziness Handicap Inventory (DHI). Both scales are discussed elsewhere in this issue of the Journal of Vestibular Research so they will be described here only briefly. Several other scales in the literature, including the UCLADQ, the DHI, and the more recent Vestibular Rehabilitation Benefit Questionnaire [23] are not ratings of in- 
dependence in activities of daily living but are scales that rate self-perceived quality of life and include one or more questions about independence in activities of daily living. Independence in activities of daily living is one of several factors that contribute to quality of life [20]. Therefore, prior to selecting a scale to be used with patients the clinician or investigator should first decide if the desired measure is independence in activities of daily living, or if it is quality of life.

The UCLA-DQ is a 5-point self-rated ordinal scale [17]. Item 3 is a global question about the ability to perform daily activities, rated on a scale from essentially no effect to dependent. The use of that single question indicates the importance of independence in activities of daily living to the quality of life, but the question is not sufficient to learn enough about which specific activities the patient has difficulty performing, and in what way performance is decreased. The UCLA-DQ is not comparable to the VADL.

The DHI is a 25-item, self-rated, 3-point ordinal scale. It has three subscales, one of which has nine items that address function. The DHI is well normed, and easy for staff and patients to use. The 3-point scale and brief list of functional skills, however, limit its value for treatment planning or even for understanding the subtleties of patients' functional limitations.

The VADL has 28 questions divided into three subgroups: functional, ambulation and instrumental skills (See Fig. 1). The 12 functional skills provide a detailed list of personal and self-care skills that are routine activities for most adults in most cultures. The nine ambulation skills describe common mobility skills for many adults in most Western cultures. Some people may not need some skills, such as using an escalator (A-21) if that person lives in a community without multistory buildings or does not enter multistory buildings that have escalators. Similarly, some ambulation skills that are common in some regions were omitted, such as walking on snow or ice. During the evaluation process the astute clinician will know to assess those skills. The Instrumental section lists seven tasks. Item I-22, driving a car, may seem redundant to Item I-28, traveling around the community. Both items are included because some communities have excellent public transportation and discourage use of individual vehicles, but some communities have minimal public transportation so most people drive their own vehicles. Also, patients with low vision or other physical limitations may be unable to drive. The VADL is available from the author in Spanish and has also been translated into Korean [22] and Portuguese [26].
Alghwiri et al. [2] pointed out that the VADL includes 41 of the 312 concepts included in the International Classification of Functioning, Disability and Health (ICF) [1]. They also pointed out that the VADL includes two concepts, transferring into and out of the bathtub or shower (Item F-7) that are not included in the ICF. In other words, the VADL added new items to formal assessment of self care skills and mobility skills beyond the standard list used by the World Health Organization.

If an individual patient does not engage in a specific activity listed in the VADL that activity may be rated as NA and should not be included in the score. Face validity was established in two ways. Patients were asked about their activities. After a preliminary list was generated some members of the Texas Occupational Therapy Association - Gulf Coast District, and other colleagues around the United States, kindly took the time to give their expert opinions about the activities listed in the scale.

The VADL uses a 10-point scale that was developed because patients who used a previous 5-point scale [11] complained that the scale was too limiting and asked for more options. The current 10-point scale, which has fine gradations at the low end of the scale, was developed from the responses of patients who subsequently received vestibular rehabilitation. Thus the scale accurately represents patients' own experiences and is useful for understanding how disabled a patient may be in reference to each particular activity or task.

Scoring is based on the ordinal or median scores that therapists often use in the clinic, such as safety guarding, and minimal and maximal assistance. The original papers reported use of the median scores for the total score and the three subscores, but subsequent work showed that the mean scores provide equivalent results [12]. Therefore the clinician may report either the median or the mean. The total score may be used or just a particular subscore, depending on the needs of the patient and the clinician.

The VADL was designed to be used during assessment of functional limitations, regardless of specific diagnosis. Indeed the VADL correlates poorly with measures of vertigo [10], does not discriminate among diagnoses [13], and does not correlate well with scores that indicate changes in sensory weighting in patients with unilateral vestibular disorders [3,10,25]. Many different disorders cause vertigo and disequilibrium. The VADL shows differences between asymptomatic, healthy controls and a heterogeneous group of patients with chronic vertigo caused by vestibular disorders as 
well as a group of patients with BPPV [10]. These findings indicate that the VADL does what it was intended to do: evaluate functional limitations regardless of the underlying pathophysiology.

Functional performance is a different level of analysis than diagnosis or specific balance skills. Functional performance may be affected by a wide variety of physical, psychological and environmental factors. Psychological needs are related to functional performance and contribute to overall quality of life [20]. Therefore the finding that VADL scores are related to performance on the Beck Depression Inventory and the Spielberger State-Trait Anxiety Inventory [16] is not surprising.

In comparison to the DHI the VADL has more possible levels for rating. The VADL and the DHI are linearly related up to level 3 of the VADL so that a 1unit change on the VADL was associated with a 20unit change on the DHI [10]. For VADL scores equal to or greater than 4 , however, the DHI scores remained the same. Thus, for greater levels of independence, i.e., levels 1 to 3 on the VADL, both scales represent selfperceived performance well. For levels that indicate less independence, however, the VADL is more sensitive than the DHI. Studies of both instruments indicate that age does not influence score $[10,18]$. This finding makes sense intuitively because age is not disabling; the disorders that occur with greater frequency with advancing age are disabling. When patients were asked to rate which tasks on the VADL were essential rather than somewhat essential or discretionary $97 \%$ of subjects rated the functional skills as essential; subjects rated the ambulation and instrumental skills as somewhat essential to discretionary. Therefore the omission of functional or personal care skills from other scales is a significant oversight that limits the value of data obtained from those scales.

The VADL is sensitive to change after relevant treatment for several different disorders. Patients with vestibular neuronitis or labyrinthitis showed improved VADL scores after vestibular rehabilitation with habituation exercises [8,9], Cawthorne exercises [22,27], balance therapy [30], and mixed habituation, gaze stabilization and balance training exercises and a walking program [28]. Patients with BPPV showed improvement on the VADL after treatment with canalith repositioning and liberatory maneuvers $[14,26]$. People with familial ataxic showed improvement on the VADL after pharmacologic treatment [29].

The scale is intended to address problems that patients with vestibular impairments often develop. It is not, however, specific to any condition, so it may be used with any patient with a vestibular weakness even if that patient has co-morbid conditions. It may even be used with patients whose main complaints are toehr health problems, because of its focus on functional performance of specific tasks, rather than for specific diagnoses. The scale has been recommended for use with patients who incur traumatic brain injury, including vestibular impairments, due to blast exposure [24].

Changes on the VADL have been shown to correlate with changes in functional magnetic resonance imaging during compensation [15]. This elegant and technically challenging study may have reached the grail of neurorehabilitation research. It is the first experiment to show that functional performance is directly related to a physiologic measure of compensation.

In summary the VADL is easy to use as a self-rating scale for patients and may also be used to structure a rehabilitation evaluation visit. It has more levels of ratings than other scales and describes more functional skills. It is sensitive to change after treatment and is related to changes in the brain caused by central mechanisms of compensation.

\section{Acknowledgements}

This paper is based on a talk given at the preconference symposium on Measures for Level of Functioning and Quality of Life in People with Vestibular Disorders, on June 10, 2102, at the XXVIIth Barany Society Meeting, Uppsala, Sweden. The symposium was sponsored by the Ad Hoc Committee on Vestibular Rehabilitation Therapy.

\section{References}

[1] International Classification of Functioning, Disability and Health, World Health Organization, Geneva, 2002.

[2] A.A. Alghwiri, G.F. Marchetti and S.L. Whitney, Content comparison of self-report measures used in vestibular rehabilitation based on the international classification of functioning, disability and health, Phys Ther 91 (2011), 346-357.

[3] F.O. Black, Clinical status of posturography in neurotology, Curr Opin Otolaryngol Head Neck Surg 9 (2001), 314-318.

[4] H. Cohen, Vestibular rehabilitation reduces functional disability, Otolaryngol Head Neck Surg 107 (1992), 638-643.

[5] H. Cohen, L.R. Ewell and H.A. Jenkins, Disability in Ménière's disease, Arch Otolaryngol Head Neck Surg 121 (1995), 29-33.

[6] H.S. Cohen and J. Jerabek, Efficacy of treatments for posterior canal benign paroxysmal positional vertigo, Laryngoscope 109 (1999), 584-590. 
[7] H.S. Cohen and K.T. Kimball, Development of the Vestibular Disorders Activities of Daily Living Scale, Arch Otolaryngol Head Neck Surg 126 (2000), 881-887.

[8] H.S. Cohen and K.T. Kimball, Increased independence and decreased vertigo after vestibular rehabilitation, Otolaryngol Head Neck Surg 128 (2003), 56-66.

[9] H.S. Cohen and K.T. Kimball, Changes in gait ataxia and balance after vestibular rehabilitation, Otolaryngology - Head and Neck Surgery 130 (2004), 418-425.

[10] H.S. Cohen, K.T. Kimball and A.D. Adams, Application of the Vestibular Disorders Activities of Daily Living Scale, Laryngoscope 110 (2000), 1204-1209.

[11] H.S. Cohen, K.T. Kimball and H.A. Jenkins, Factors affecting recovery after acoustic neuroma resection, Acta Otolaryngologica 122 (2002), 841-850.

[12] H.S. Cohen and H. Sangi-Haghpeykar, Canalith repositioning variations for benign paroxysmal positional vertigo, Otolaryngol Head Neck Surg 143 (2010), 405-412.

[13] M. Duracinsky, I. Mosnier, D. Bouccara, O. Sterkers, O. Chassany and W.G.o.t.S.F.d.O.-R.-L. (ORL), Literature review of questionnaires assessing vertigo and dizziness, and their impact on patients' quaity of life, Value Health $\mathbf{1 0}$ (2007), 273-284.

[14] E.A. Guneri and O. Kustutan, The effects of betahistine in addition to eEpley maneuver in posterior canal benign paroxysmal positional vertigo, Otolaryngol Head Neck Surg 46 (2012), 104-108.

[15] C. Helmchen, J.C. Klinkenstein, A. Krüger, J. Gliemroth, C. Mohr and T. Sander, Structural brain changes following peripheral vestibulo-cochlear lesion may indicate multisensory compensation, J Neurol Neurosurg Psychiatr 82 (2011), 309_ 316.

[16] S.M. Hong, B.G. Kim, B.C. Lee, S.K. Park, S.K. Hong, H.J Lee, H.J. Kim, J.H. Lee, C.W. Kim, I.S. Park and Y.B. Kim, Analysis of psychological distress after management of dizziness in old patients: Multicenter study, Eur Arch Otorhinolaryngol 269 (2012), 39-43.

[17] V. Honrubia, T.S. Bell, M.R. Harris, R.W. Baloh and L.M. Fisher, Quantitative evaluation of dizziness characteristics and impact on quality of life, Amer J Otol 17 (1996), 595-602.

[18] G.P. Jacobson and C.W. Newman, The development of the Dizziness Handicap Inventory, Arch Otolaryngol Head Neck Surg 116 (1990), 424-427.
[19] R.M. Klein and B. Bell, Self-care skills: Behavioral measurements with the Klein-Bell ADL Scale, Arch Phys Med Rehabil 63 (1982), 335-338

[20] H.J. Lee and S. Choi-Kwon, Quality of life and the related factors in patients with dizziness, J Korean Acad Nurs 39 (2009), 751-758.

[21] F.I. Mahoney and D.W. Barthel, Functional evaluation: the Barthel Index, Md Med J 14 (1965), 61-65.

[22] K.K. Min, M.J. Ha, C.H. Cho, H.E. Cha and J.H. Lee, Clinical use of subjective visual horizontal and vertical in patients of unilateral vestibular neuritis, Otol Neurotol 28 (2007), 520524.

[23] A.E. Morris, M.E. Lutman and L. Yardley, Measuring outcome from vestibular rehabilitation, part I: Qualitative development of a new self-report measure, Int J Audiol 47 (2008), 169-177.

[24] P.J. Myers, D.J. Wilmington, F.J. Gallun, J.A. Henry and S.A. Fausti, Hearing impairment and traumatic brain injury among soldiers: Special considerations for the audiologist, Seminars in Hearing 30 (2009), 5-27.

[25] R.J. Peterka, K.D. Statler, D.M. Wrisley and F.B. Horak, Postural compensation for unilateral vestibular loss, Front Neurol 2 (2011), 57.

[26] C.R. Resende, C.K. Taguchi, J.G. de Almeida and R.R. Fujita, Vestibular rehabilitation in elderly patients with benign paroxysmal positional vertigo, Rev Bras Otorrinolaringol 69 (2003).

[27] F. Salvinelli, M. Casale, M. Trivelli, L. D’Ascanio, L. Firrisi, F. Lamanna, F. Greco and S. Costantino, Benign paroxysmal positional vertigo: A comparative prospective study on the efficacy of Semont's maneuver and no treatment strategy, $\mathrm{La}$ Clinica terapeutica 154 (2003), 7-11.

[28] P.S. Shah and J.S. Kale, A study of the effects of a vestibular rehabilitation program on patients with peripheral vestibular dysfunctions, Indian J Occup Ther 36 (2004), 11-16.

[29] M. Strupp, R. Kalla, J. Claassen, C. Adrion, U. Mansmann, T. Klopstock, T. Freilinger, H. Neugebauer, R. Spiegel, M. Dichgans, F. Lehmann-Horn, K. Jurkat-Rott, T. Brandt, J.C. Jen and K. Jahn, A randomized trial of 4-aminopyridine in EA2 and related familial episodic ataxias, Neurology 77 (2011), 269-275.

[30] D.M. Wrisley and M.J. Stephens, The effects of rotational platform training on balance and ADLs, in: Annual International Conference of the IEEE Engineerng in Medicine and Biology Society, vol 2011, 2011, pp. 3529-3532. 


\section{Vestibular Disorders Activities of Daily Living Scale}

Name

Data

\section{Instructions}

This scale evaluates the effect or vertigo and balance disorders on independence in routine activities of daily living. Please rate your performance on each item. If your performance varies due to intermittent dizziness or balance problems please use the greatest level of disability. For each task indicate the level which most accurately describes how you perform the task. If you never do a particular task, please check the box in the column NA. The rating scales are explained on the bottom of the page.

\begin{tabular}{|c|c|c|c|c|c|c|c|c|c|c|c|}
\hline \multirow{2}{*}{ Task } & \multicolumn{11}{|c|}{ Independence rating } \\
\hline & 1 & 2 & 3 & 4 & 5 & 6 & 7 & 8 & 9 & 10 & $\overline{\mathrm{NA}}$ \\
\hline \multicolumn{12}{|l|}{ F-1 Sitting up from lying down } \\
\hline \multicolumn{12}{|l|}{ F-2 Standing up from sitting on the bed or chair } \\
\hline \multicolumn{12}{|l|}{ F-3 Dressing the upper body (e.g. shirt, brassiere, undershirt) } \\
\hline \multicolumn{12}{|l|}{ F-4 Dressing the lower body (e.g. pants, skirt, underpants) } \\
\hline \multicolumn{12}{|l|}{ F-5 Putting on socks/ stockings } \\
\hline \multicolumn{12}{|l|}{ F-6 Putting on shoes } \\
\hline \multicolumn{12}{|l|}{ F-7 Moving in/ our of the bathtub or shower } \\
\hline \multicolumn{12}{|l|}{ F-8 Bathing yourself in the bathtub or shower } \\
\hline \multicolumn{12}{|l|}{ F-9 Reaching overhead (e.g. to a cupboard or shelf) } \\
\hline \multicolumn{12}{|l|}{ F-10 Reaching down (e.g. to the floor or a shelf) } \\
\hline \multicolumn{12}{|l|}{ F-11 Meal preparation } \\
\hline \multicolumn{12}{|l|}{ F-12 Intimate activity (e.g. foreplay, sexual activity) } \\
\hline \multicolumn{12}{|l|}{ A-13 Walking on level surfaces } \\
\hline \multicolumn{12}{|l|}{ A-14 Walking on uneven surfaces } \\
\hline \multicolumn{12}{|l|}{ A-15 Going up steps } \\
\hline \multicolumn{12}{|l|}{ A-16 Going down steps } \\
\hline \multicolumn{12}{|l|}{ A-17 Walking in narrow spaces (e.g. corridor, grocery store aisle) } \\
\hline \multicolumn{12}{|l|}{ A-18 Walking in open spaces } \\
\hline \multicolumn{12}{|l|}{ A-19 Walking in crowds } \\
\hline \multicolumn{12}{|l|}{ A-20 Using an elevator } \\
\hline \multicolumn{12}{|l|}{ A-21 Using an escalator } \\
\hline \multicolumn{12}{|l|}{ I-22 Driving a car } \\
\hline \multicolumn{12}{|l|}{ I-23 Carrying things while walking (e.g. package, garbage bag) } \\
\hline \multicolumn{12}{|l|}{ I-24 Light household chores (e.g. dusting, putting items away } \\
\hline \multicolumn{12}{|l|}{ I-25 Heavy household chores (e.g. vacuuming, moving furniture) } \\
\hline \multicolumn{12}{|l|}{ I-26 Active recreation (e.g. sports, gardening) } \\
\hline I-27 Occupational role (e.g. job, child care, homemaking, student) & & & & & & & & & & & \\
\hline I-28 Traveling around the community (e.g. car, bus) & & & & & & & & & & & \\
\hline
\end{tabular}

The VADL scale [7]. (Copyright @ 2000 American Medical Association. All rights reserved.)

\section{Explanation of Independence Rating Scale}

This scale will help us to determine how inner ear problems affect your ability to perform each task. Please indicate your current performance on each task, as compared to your performance before developing an inner ear problem, by checking one of the columns in the center of the page. Pick the answer that most accurately describes how you perform the task.

1. Independent. I am not disabled. I perceive no change in performance from before developing in inner ear impairment.

2. I am uncomfortable performing the activity but perceive no difference in the quality of my performance.

3. Decreased ability, no change in manner of performance. I perceive a decrement in the quality of my performance but have not changed the manner of my performance. 
4. Slower, cautious, more careful. I have changed the manner of my performance, i.e., I do things more slowly or carefully than before, or I do things without bending.

5. Prefer using an object for help. I prefer using an ordinary object in the environment for assistance (e.g., stair railing) but I am not dependent on the object or device to do the activity.

6. Must use an object for help. I must use an ordinary object in the environment for assistance, but I have not acquired a device specifically designed for the particular activity.

7. Must use special equipment. I must use adaptive equipment designed for the particular activity (e.g., grab bars, cane, reachers, bus with lift, wedge pillow).

8. Need special assistance. I require another person for physical assistance or, for an activity involving two people I need unusual physical assistance.

9. Dependent. I am dependent on another person to perform the activity.

10. Too difficult, no longer perform. I no longer perform the activity due to vertigo or a balance problem.

11. NA. I do not usually perform this activity or I prefer not to answer this question. 